\section{A Checklist in the Event of Aneurysm Perforation during Coiling}

The use of checklists is well-established in aviation, where their application is an integral part of every sequence of flying. ${ }^{1}$ In cases of emergency flying procedures, the use of checklists is fundamental in dealing with critical tasks, and their application is a central part of pilot training and pilot performance. ${ }^{2}$

Checklists have only recently been introduced in medicine in an effort to manage a number of procedures in a uniform manner, according to the established standard of care. ${ }^{3}$ While suited especially for complex or emergency tasks, the application of a checklist in reducing morbidity and mortality for the placement of a central line has gained wide recognition. ${ }^{4-7}$

Recently, Atul Gawande, a surgeon at Harvard Medical School, has published his experience with checklists in medicine, aviation, and building construction, in his book The Checklist Manifesto: How to Get Things Right. ${ }^{8}$ The book details his experience in developing a checklist for the World Health Organization in its global effort to reduce the morbidity and mortality of surgical interventions. The checklist incorporated 19 simple items, ranging from the identification of the correct patient and correct surgical site to confirming the application of preoperative antibiotics and making sure all team members in the operating room know each other and their respective roles. ${ }^{8,9}$ Their initial results, published in the New England Journal of Medicine in 2009, showed dramatic results in reducing both morbidity and mortality at the pilot study sites. ${ }^{9}$

This effort has led us to believe that checklists have the potential of becoming significant tools in making medicine, and more specifically interventions, safer. ${ }^{3}$ From a neurointerventional point of view, it was the belief the authors, comprising both neurointerventionalists (G.L., H.C., D.K.) and neurosurgeons (Ph.T., G.L.), that the perforation of aneurysms during coiling is one of the most critical events in the neurointerventional field. The correct response in these instances is guided not only by experience but also by taking the correct measures, with a large number of steps involving clinical, interventional, anesthesiologic, and organizational aspects. As a result, a checklist may help both the experienced and less experienced practitioner to react in the event of aneurysmal rupture according to the best standard of care. $^{10}$
The checklist (Fig 1) proposed here is an initial effort to translate the personal and published experience of both neurosurgeons and neuroradiologists regarding the handling of an aneurysmal perforation during coiling according to the best standard of care. At the heart of this effort is the inspiration of Atul Gawande's book, and we are eager to have the checklist used, studied, and improved.

\section{References}

1. Degani A, Wiener EL. Procedures in complex systems: the airline cockpit. IEEE Trans Syst Man Cybern A Syst Hum 1997;27:302-12

2. Rantz WG, Dickinson AM, Sinclair GA, et al. The effect of feedback on the accuracy of checklist completion during instrument flight training. J Appl Behav Anal 2009;42:497-509

3. Winters BD, Gurses AP, Lehmann $\mathrm{H}$, et al. Clinical review: checklists-translating evidence into practice. Crit Care 2009;13:210. Epub 2009 Dec 31

4. Pronovost PJ, Goeschel CA, Colantuoni E, et al. Sustaining reductions in catheter-related bloodstream infections in Michigan intensive care units: observational study. BMJ 2010;340:c309

5. Watson SR, George C, Martin M, et al. Preventing central line-associated bloodstream infections and improving safety culture: a statewide experience. Jt Comm J Qual Patient Saf 2009;35:593-97

6. Hales BM, Pronovost PJ. The checklist: a tool for error management and performance improvement. J Crit Care 2006;21:231-5

7. Berenholtz SM, Pronovost PJ, Lipsett PA, et al. Eliminating catheter-related bloodstream infections in the intensive care unit. Crit Care Med 2004;32: 2014-20

8. Gawande A. The Checklist Manifesto: How to Get Things Right. New York: Metropolitan Books; 2009

9. Haynes AB, Weiser TG, Berry WR, et al, for the Safe Surgery Saves Lives Study Group. A surgical safety checklist to reduce morbidity and mortality in a global population. $N$ Engl J Med 2009;360:491-99. Epub 2009 Jan 14

10. Doerfler A, Wanke I, Egelhof T, et al. Aneurysmal rupture during embolization with Guglielmi detachable coils: causes, management, and outcome. AJNR Am J Neuroradiol 2001;22:1825-32

Ph. Taussky

Department of Neurosurgery

University of Utah

Salt Lake City, Utah

G. Lanzino

H. Cloft

D. Kallmes

Department of Radiology and Neurosurgery

Mayo Clinic College of Medicine

Rochester, Minnesota

DOI 10.3174/ajnr.A2140

\begin{tabular}{|l|}
\hline IDENTIFICATION OF PERFORATION \\
Owire/coil beyond aneurysm edge \\
Alert Anesthesia about perforation \\
Do not retract wire/catheter/coil \\
Perform angiography to look for \\
extravasation \\
Look at transit time \\
Consider CT now/after \\
cLINICAL EXAM \\
Pupil status \\
Glasgow Coma Scale \\
Blood Pressure Change \\
Focal neurologic deficit \\
Agitation
\end{tabular}
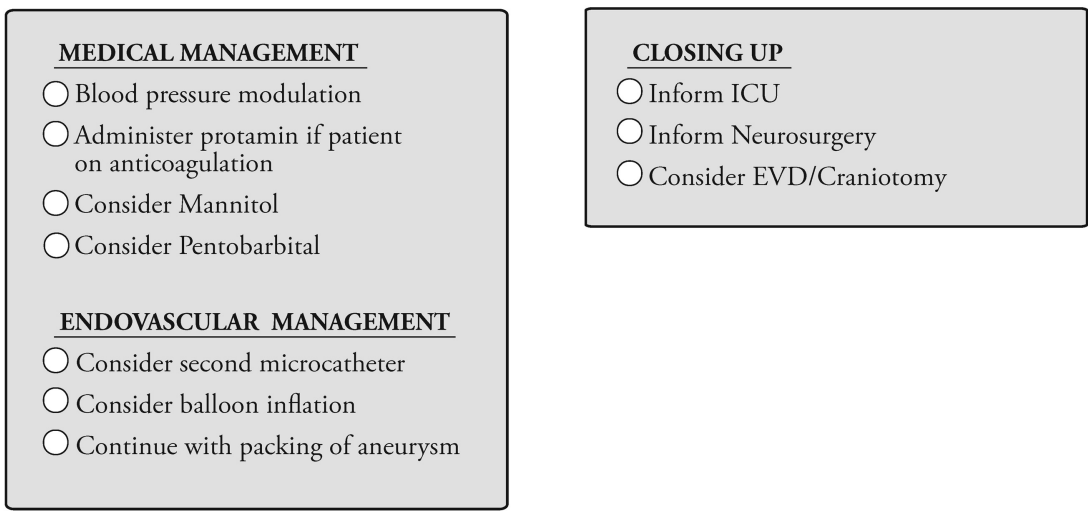

Fig 1. Checklist for aneurysm perforation during coiling. ICU indicates intensive care unit; EVD, external ventricular drain. 